\title{
IMPLEMENTING CLINICAL TRIALS IN ONCOLOGY WITH QUALITY OF LIFE ASSESSMENT AND PATIENT REPORTED OUTCOMES. AN INTERVIEW TO EXPLORE THE ROAD TOWARDS A MORE PATIENT-CENTERED APPROACH
}

\author{
M. Di Maio \\ Department of Oncology, University of Turin, Turin, Italy \\ Division of Medical Oncology, Ordine Mauriziano Hospital, via Magellano 1, Turin 10128, Italy
}

\section{CORRESPONDENCE TO:}

E-mail: massimo.dimaio@unito.it

Twitter account: @MassimoDiMaio75

ORCID: 0000-0001-8906-3785

Doi: $10.48286 / a r o .2021 .19$

Health-related quality of life (QoL) is universally considered a measure of clinical benefit for patients with cancer, but its inclusion in clinical trials has historically been suboptimal. The recognition of the role of QoL and patient-reported outcomes in the evaluation of new agents by regulatory agencies and in the definition of treatment value is constantly increasing. ARO editors asked Prof. Massimo Di Maio, from the Department of Oncology, University of Turin and National Secretary of the Italian Association of Medical Oncology, critical questions about the use of patient-reported outcomes in clinical trials conducted in Oncology.

$\mathrm{M}$ assimo Di Maio is associate Professor of Medical Oncology at Department of Oncology, University of Turin, Italy, since 2014, and director of Medical Oncology at Mauriziano Hospital, Turin, since 2016. In 1999, he obtained degree cum laude in Medicine and Surgery at the Federico II University of Naples, Italy. In 2003, he graduated from the Specialty School in Oncology at the same University. From 2000 to 2006 and from 2008 to 2014 he worked at the Clinical Trials Unit of the National Cancer Institute G. Pascale Foundation, in Naples, Italy, where he was involved in the planning, conducting and analysis of clinical trials. His main areas of interest are the methodology of clinical trials and meta-analyses in Oncology and the use of patient-reported outcomes in clinical research and in clinical practice. Prof. Di Maio has been invited as speaker at many national and international meetings and has authored more than 300 publications in international peer-reviewed journals (H-Index April 2021: 45 according to Scopus). Additionally, Prof. Di Maio is an active member of the Associazione Italiana di Oncologia Medica (Italian Association of Medical Oncology, AIOM) and the European Society of Medical Oncology (ESMO). Between 2009 and 2013, Prof. Di Maio acted as national coordinator of the AIOM Young Oncologists Working Group. Between 2013 and 2017, he was member of AIOM National Board and since October 2019 he is AIOM National Secretary. 
AOR:

In the last years, you published several studies pointing to the need to include quality of life (QoL) among the endpoints of clinical trials conducted in oncology. Why this choice?

\section{Massimo Di Maio:}

As a medical oncologist, I strongly believe that QoL and patient-reported outcomes have a crucial role both in clinical trials and in clinical practice. In 2017, we started working to a systematic review about the adoption of QoL in cancer clinical trials (1). I was convinced that such an analysis could have been important in denouncing, to the scientific community, the lack of attention to the QoL of patients in the development and approval of new treatments which, hopefully, can become new standard therapies. In addition, this kind of work, implying the screening and careful analysis of many hundreds of publications, could have been carried out only counting on the collaboration of many colleagues, and so I involved a number of young fellows attending the Specialty School in Oncology. All of them were enthusiast about the study. The added value of that experience, in my view, has been precisely to raise awareness on the topic, not only among the scientific community (once our paper has been published and discussed), but particularly among all the young colleagues who have been involved in the work. I am pleased to think that attention to quality of life and patient-reported outcomes for patients with cancer has been part of their training.

AOR:

What is the definition of patient-reported outcomes and what is their added value to clinical research?

\section{Massimo Di Maio:}

Any outcome evaluated directly by the patient and based on patient's perception of a disease and its treatment is called patient-reported outcome (PRO). From this "general" definition, it is clear that the term PRO is an umbrella term, which can cover measures of symptoms, health-related quality of life, health status, adherence to treatment, satisfaction with treatment, and many other important domains. No need to specify that these instruments have a relevant role in oncology. Not only from a regulatory point of view, but also from a clinical point of view, the main goal of any anticancer treatment is to allow patients to live longer and/or to live better, so the point of view of patients is essential to evaluate the efficacy of new anticancer treatments. In recent years, progression-free survival (PFS) has been often adopted as primary endpoint in many trials, instead of overall survival (OS). When the experimental treatment demonstrates a benefit in PFS (that means in the instrumental control of the disease), PROs and QoL measures are particularly important to better define the real clinical impact of a treatment. However, I believe that even when the experimental treatment demonstrates a clinically relevant improvement in OS, PROs and QoL results are not useless and are still of interest, because they allow a more complete definition of benefits and harms associated with the treatment. I still think that in the era of precision medicine, the selection of the right drug for the right patient cannot neglect the opinion of the patients regarding what can be considered really right for themselves. I think that this is a fundamental part of a good tailored medicine.

AOR:

If you had only 5 minutes to convince investigators who are designing a clinical trial to include patient-reported outcomes among the endpoints, what are the main reasons you would emphasize?

\section{Massimo Di Maio:}

They should be the ones to convince me why NOT including PROs in their trial. Seriously, European Medicines Agency (EMA) helps me to provide this answer (2). EMA has provided a convincing list of reasons to include patient-reported outcomes in cancer clinical trials, and I personally agree with all of those 5 reasons. Firstly, PROs can provide a patient focused assessment of the burden and impact of disease, by understanding how a treatment impacts on patient functioning and well-being. This means "giving voice to the patient", not rhetorically but substantially. Second, their analysis can add information on the clinical benefit of a therapy by complementing efficacy and safety data with patient-reported evaluation. Thirdly, PROs allow assessing the relationship/ agreement between clinical reported endpoints and patient-reported endpoints (and we have shown that symptoms and adverse events can be significantly underreported by clinicians). Fourth, PROs are essential in the non-inferiority trial setting: in this kind of trials, we accept to "sacrifice" part of the efficacy 
obtained with the standard treatment, at the condition that the experimental treatment is preferable to the patient, so the measure of quality of life is essential to verify this assumption. Last but not least, in the clinical practice following the approval of the treatment, results of PROs obtained in the clinical trial can provide important information to facilitate more accurate future patient-physician communication in terms of the quality of life for the patient and the burden of treatment-related morbidities and disease-related patient impacts.

\section{AOR:}

\section{How can QoL be formally included in the evalu- ation of treatment value?}

\section{Massimo Di Maio:}

In recent years, stimulated by the debate about the rising prices of cancer drugs and about the flickering sustainability of the health system, scientific societies have tried to produce some instruments for the formal evaluation of treatment value. For me, it is not surprising that both the framework proposed by the American Society of Clinical Oncology (ASCO) and the European Society of Medical Oncology (ESMO) magnitude of clinical benefit scale (MCBS) evaluating the value of anticancer treatments include QoL results among the parameters considered for the evaluation of study results. Briefly, in the ASCO framework a "palliation bonus" is awarded by the experimental treatment, if a statistically significant improvement in cancer-related symptoms is shown, and a "QoL bonus" is awarded if a statistically significant improvement in QoL is demonstrated (3). Similarly, in the ESMO MCBS, preliminary scores based on treatment efficacy can be upgraded when the experimental treatment demonstrates improved QoL or delayed deterioration in QoL (or substantial reduction in severe toxicity) (4). Consequently, there is no doubt that a complete evaluation of treatment value can be properly made only if the scientific community could evaluate QoL results at the same time of the other endpoints of a trial.

Beyond the attention demonstrated by scientific societies, also regulatory agencies have made explicit their attention to QoL and PROs. Namely, both EMA and FDA have produced documents dedicated to the inclusion of PROs and quality of life in cancer clinical trials. These documents can easily be found on the web, and I believe this would be an interesting lecture for oncologists $(2,5)$. From my point of view, it means that regulatory agencies declare they will pay attention to these endpoints in the evaluation of treatments for the authorization for use in clinical practice. Until recent years, we must admit that this was not the case, at least for the vast majority of treatments: several analyses have shown that only a small proportion of cancer drugs approved for use in clinical practice have demonstrated a significant improvement in patients' quality of life (6).

\section{AOR:}

You emphasize the importance of inclusion of patient-reported outcomes and QoL in clinical trials. However, this implies that investigators, and readers, become more familiar with specific methodology of analysis and presentation of results. Which are the most relevant issues in the methodology of QoL analysis?

\section{Massimo Di Maio:}

I have been involved in the analysis of QoL data for several clinical trials, and of course I am aware that the adoption of QoL among endpoints implies several methodological issues (1). First of all, when designing the trial and planning the study procedures, the choice of the correct QoL questionnaires and the best timing of administration are crucial. During the trial, all the efforts should be made to optimize the compliance to questionnaires (which depends not only on the patients' motivation but especially on the attention paid by investigators): missing data can be a relevant problem, especially in diseases such as advanced lung cancer or pancreatic cancer or other advanced tumors characterized by a dismal prognosis, where the proportion of early treatment failures is often unfortunately not negligible. This can be a problem for the analysis, especially if the proportion of missing patients is unbalanced among study arms, considering that patients who do not fill in the questionnaires are mostly those who are getting worse (this phenomenon is called "missing not at random"), so we risk to catch a picture of patients' quality of life that can be biased compared to the whole study population. Furthermore, as we have repeatedly shown in our analyses conducted in lung cancer (7), or in prostate cancer (8), or in colorectal cancer (9), methodology of analysis and presentation of results in the publications can be really heterogeneous. Many publications present mean scores at different time points or mean changes compared to baseline, but this analysis (which is useful to compare the "global" trends of different study arms), does not allow to understand how many patients 
experienced a significant improvement (or a significant worsening) of quality of life. The latter information is better evaluable describing the proportion of responders, which is, however, presented only in a minority of papers. In addition, time to deterioration of specific symptoms or of global QoL (which allows the production of curves that are "familiar" to readers, being similar to Kaplan Meier curves of overall survival or progression-free survival) is particularly useful to focus on treatment failure, helping to define if a radiologically defined benefit (like progression-free survival) is sustained by a subjective benefit. Whichever the modality of presentation of results, as for the other endpoints of treatment efficacy, when reading and interpreting QoL data, clinical relevance of the difference between arms should always be considered in addition to statistical significance. In addition to the presentation of results, all papers should explicit and reference the definition of minimum clinically important difference for all the QoL scores included in the analysis (10).

\section{AOR:}

In your analysis describing the deficiencies of QoL inclusion in cancer clinical trials, you found that many randomized trials did not include QoL among endpoints. Furthermore, you emphasized that, even in trials including QoL among endpoints, there was often a delay in the publication of QoL results, which are often not included in the primary study publication. How do you comment that finding?

\section{Massimo Di Maio:}

Yes, we found that, in a significant proportion of trials, QoL results were not included in the primary publication and there is a delay (of months, sometimes even of years) in the publication of QoL results (1). Many colleagues - both as investigators and as readers - are accustomed to this, and consider it normal that quality of life data should be presented at congress and published abundantly after the main publication. To be honest, we were really disappointed of this. If you consider that, by definition, QoL data are available at the moment of primary analysis (because they are collected during the treatment), it is a real scientific shame that QoL results are often not included in the primary publication. Although the results from negative trials are important for the scientific community, we admit that the publication of QoL results of negative trials could be considered less essential, because those trials will not imply the subsequent adoption of experimental treatments in clinical practice. On the other hand, there is no doubt that the absence of QoL data is particularly disappointing for trials with formally positive results. In fact, if the experimental treatment has shown a benefit in terms of OS, QoL results are helpful to define the trade-off between benefits and harms, helping to define the value, especially if the survival benefit is modest and the potential toxicity of the treatment is not negligible. QoL results are even more important when the interpretation of the trial is based on a surrogate endpoint (such as PFS): in this case, we strongly believe that a patient-focused assessment should be crucial to define the value of a radiologically defined result. However, when discussing with Laura Marandino, Franco Perrone and all the other authors involved in our work, we have asked ourselves why QoL results are often published later. There are several potential reasons: journals impose limitations of article length, and QoL results typically need much space to be adequately described, so a second publication appears a good solution. However, I believe that, in the era of electronic publications, the number of article pages should no more be a problem, and all the material that the authors consider relevant could be published as supplementary material. Another issue could be the willingness of producing more than one publication for the same trial, also to allow visibility for a higher number of authors. My proposal, in this case, would be to submit the QoL paper as a simultaneous, companion paper to the same journal, and let the editor decide.

There is a general agreement that publication of QoL results is relevant, and I strongly believe that their publication should be available along with the other trial endpoints. In this sense, an effort by authors and editors for a complete and timely reporting of clinical trials, including all relevant outcomes, would allow an exhaustive evaluation of the bene$\mathrm{fit} /$ risk ratio and of the value of a new treatment.

AOR:

In conclusion, what do you think will be the role of QoL and patient-reported outcomes in the years to come?

\section{Massimo Di Maio:}

In the editorial accompanying our 2018 paper in the Annals of Oncology, Lesley Fallowfield defined QoL a "Cinderella" outcome (10). I found that definition very fitting, because QoL has traditionally received subop- 
timal attention, even in the setting of advanced cancer, where the attention to patients' symptoms and to the burden of treatment toxicity should be very high. QoL has often been considered a topic for experts in the field, an interesting but all in all "superfluous" endpoint. From this point of view, I am glad to see that the importance of QoL results in the definition of treatment value, within a patient-centered approach, is definitely increasing. I really hope that, if we will repeat our previous analysis comparing the adoption of QoL in trials published in the last 5 years with the results we observed in the period 2012-2016, we will observe a significant improvement in the proportion of clinical trials adopting QoL and presenting results in the publication. And let's be optimistic and believe that the results in the future 5 years will be even better. As I have repeatedly said in these years, all stakeholders (patients, researchers, sponsors, regulatory agencies and scientific journals) should encourage the inclusion of QoL among study endpoints, with a complete and timely reporting of QoL results in the publications.

\section{ETHICS}

\section{Fundings}

There were no institutional or private fundings for this article.

\section{Conflict of interests}

The authors have declared no conflict of interests.

\section{Availability of data and material}

N/A

\section{Authors' contribution}

MDM was involved in drafting the manuscript and revising it critically for important intellectual content, and gave final approval of the version to be published.

\section{Ethical approval and consent to participate} N/A 


\section{REFERENCES}

1. Marandino L, La Salvia A, Sonetto C, et al. Deficiencies in health-related quality-of-life assessment and reporting: a systematic review of oncology randomized phase III trials published between 2012 and 2016. Ann Oncol 2018;29:2288-95.

2. European Medicines Agency. EMA/CHMP/292464/ 2014 Committee for Medicinal Products for Human Use (CHMP). Appendix 2 to the guideline on the evaluation of anticancer medicinal products in man. The use of patient-reported outcome (PRO) measures in oncology studies. April 1, 2016. Available at: http://www.ema.europa.eu/docs/en_GB/document_library/Other/2016/04/WC500205159.pdf. Last accessed: May 1, 2021.

3. Schnipper LE, Davidson NE, Wollins DS, et al. Updating the American Society of Clinical Oncology Value Framework: Revisions and Reflections in Response to Comments Received. J Clin Oncol 2016;34:2925-34.

4. Cherny NI, Dafni U, Bogaerts J, et al. ESMO-Magnitude of Clinical Benefit Scale version 1.1. Ann Oncol 2017;28:2340-66.

5. US Food and Drug Administration. Patient-Reported Outcome Measures: Use in Medical Product Development to Support Labeling Claims. Guidance for Industry2009. Availableat: https://www.fda.gov/regulatory-information/ search-fda-guidance-documents/patient-reported-outcome-measures-use-medical-product-development-support-labeling-claims. Last accessed: May 1, 2021.

6. Davis C, Naci H, Gurpinar E, Poplavska E, Pinto A, Aggarwal A. Availability of evidence of benefits on overall survival and quality of life of cancer drugs approved by European Medicines Agency: retrospective cohort study of drug approvals 2009-13. BMJ 2017;359:j4530.

7. Marandino L, De Luca E, Zichi C, et al. Quality-of-Life Assessment and Reporting in Prostate Cancer: Systematic Review of Phase 3 Trials Testing Anticancer Drugs Published Between 2012 and 2018. Clin Genitourin Cancer 2019;17(5):332-47.e2.

8. Reale ML, De Luca E, Lombardi P, et al. Quality of life analysis in lung cancer: A systematic review of phase III trials published between 2012 and 2018. Lung Cancer 2020;139:47-54.

9. Lombardi P, Marandino L, De Luca E, et al. Quality of life assessment and reporting in colorectal cancer: A systematic review of phase III trials published between 2012 and 2018. Crit Rev Oncol Hematol 2020;146:102877.

10. Fallowfield LJ. Quality of life assessment using patient-reported outcome (PRO) measures: still a Cinderella outcome? Ann Oncol 2018;29:2286-7. 